\title{
Lusotopie
}

Recherches politiques internationales sur les espaces

issus de l'histoire et de la colonisation portugaises

$\mathrm{XV}(2) \mid 2008$

Histoires d'Asie

\section{Grandes espérances, mince innocence}

\section{René Pélissier}

URL : http://journals.openedition.org/lusotopie/659

ISSN : 1768-3084

Éditeur :

Association des rechercheurs de la revue Lusotopie, Brill, Karthala

Édition imprimée

Date de publication : 20 novembre 2008

Pagination : 235-246

ISSN : 1257-0273

\section{Référence électronique}

René Pélissier, «Grandes espérances, mince innocence », Lusotopie [En ligne], XV(2) | 2008, mis en ligne le 01 février 2016, consulté le 26 avril 2019. URL : http://journals.openedition.org/lusotopie/659 


\section{GRANDES ESPÉRANGES, MINGE INNOGENGE}

Combien de lecteurs un critique doit-il mécontenter ? Et faut-il même en mécontenter un seul si l'on veut survivre dans cette activité ? Et que dire des auteurs? N'avançons pas de chiffres, ne nous lançons pas dans des prévisions sur la réception de nos commentaires et, les yeux fermés ou plutôt ouverts, affirmons que les actes réunis par Ana Maria Binet ${ }^{1}$ contiennent, sous la plume de Michel Cahen, une contribution, «Lusitanité et lusophonie : considérations conceptuelles sur des réalités sociales et politiques", importante mais qui risque de déplaire fortement, non seulement aux hommes de pouvoir à Lisbonne, mais aussi aux opiomanes qui abondent encore dans la population portugaise lettrée et également - et parfois avec virulence - parmi les descendants de l'émigration. La Communauté des pays de langue portugaise (CPLP)? Un imaginaire colonial vivace (dixit p. 129), selon lui. Sans complaisance aucune pour les politiques linguistiques officielles, il insiste sur le décalage entre les rêves et les réalités, sur la flatteuse immortalité du lusotropicalisme et de ses avatars actuels. Décapant jusqu'à l'os, il assène quelques douloureuses vérités. Dans le fond, mieux vaut être professeur de littérature que politologue si l'on veut dormir tranquille dans le lit de la lusologie.

Les historiens sont-ils à l'abri des insomnies? Cela dépend des pays et des siècles dont ils parlent. Les prudents ne devraient pas dépasser le $\mathrm{xVII}^{\mathrm{e}}$ siècle et, même là, il est préférable de ne pas s'attarder sur des sujets qui fâchent. Les plus malins s'en tiendront donc à des sujets à la mode... en attendant qu'elle se démode. Dans le très riche recueil présenté par Philip J. Havik et Malyn Newitt², les études sur le rôle des femmes, les lançados en Afrique occidentale, le débat sur l'origine des Angolares à São Tomé, la famille des Bezerras sur la frontière commerciale de l'Angola, le Brésil à la fin de la période coloniale, les pirates à Madagascar (nous n'avons pas bien compris en quoi ils relèvent du monde lusophone), etc., devraient éviter à leurs auteurs des prises excessives de somnifères. En revanche, toucher à la "pureté du sang » en Angola jusqu'en 1912 ou à la présence portugaise à Goa, vue par le très regretté Orlando Ribeiro, n'est pas un gage de sérénité nocturne. Mais comme le livre est offert à la mémoire du géant que fut Charles Boxer - qui me fit l'honneur de m'inviter à déjeuner à son Army E Navy Club, en la lointaine année 1965 - le souvenir que je garde de ce grand bonhomme me confirme dans mon appréciation largement positive quant au bien-fondé de la publication - tardive - de ce texte. Ce n'est pas tous les jours que l'on a la chance de fêter le centenaire d'un être d'exception dans les études luso-impériales, alors que les livres d'hommages à des arrivistes médiocres s'accumulent dans certaines académies de Trissotins.

${ }^{1}$ A.M. Binet (ed.), Mythes et mémoire collective dans la culture lusophone, Pessac, Presses universitaires de Bordeaux, 2007, 148 p. («Eidôlon », 78).

${ }^{2} \mathrm{Ph} . J$. Havik \& M. Newitt (eds), Creole Societies in the Portuguese Colonial Empire, Bristol, University of Bristol, Department of Hispanic, Portuguese and Latin American Studies, 2007, 276 p. 
Tardive ou pas, la réception simultanée de trois livraisons des Cadernos de Estudos Africanos $^{3}$ nous montre au moins la vigueur de certains secteurs de la recherche africaniste en sciences sociales au Portugal. Elle tient même du miracle quand on se rappelle ce qu'elle était sous l'Estado Novo, le marécage où elle agonisait dans les années 1974-1986 et l'indifférence - voire l'hostilité - de certains milieux actuels. Sans parler, évidemment, de la course désespérée aux subventions nécessaires pour la faire vivre. Le $\mathrm{n}^{\circ} 5 / 6(2003-2004)$ est consacré à la politique en Afrique. On y relève, entre autres, deux articles sur le Mozambique. Le $\mathrm{n}^{\circ}$ 7/8 (2004-2005) nous enseigne plein de choses sur l'Angola et l'anthropologie physique à l'époque coloniale. C'est cependant peut-être le n 9/10 (2005-2006) qui, portant sur les mémoires coloniales, est le plus perturbant, car il analyse finement la persistance de la nostalgie impériale dans la mentalité portugaise, et même sa remontée spectaculaire comme en témoigne le succès littéraire du roman Equador. Personnellement, c'est dans le dernier article sur les prémices du nationalisme en Guinée-Bissau - et notamment le «massacre de Pidjiguiti »- qu'un nouveau pan de mes illusions s'effondre. Il ne m'en restait plus beaucoup pourtant, mais découvrir que l'historien de la traite négrière António Carreira était alors " gerente da Casa Gouveia" et qu'il fut directement impliqué dans l'affaire, renforce mon impression que, dans l'étude de l'Histoire contemporaine, on entre les mains parfois propres, mais qu'elles en ressortent souvent sales. L'innocence ne dure pas longtemps dans ces lits-là. Quant à la naïveté...

Innocence et naïveté les trouvera-t-on sous la même couverture de Passagens para África ${ }^{4}$ de Claúdia Castelo? Difficilement, car une thèse de doctorat en sociologie historique, ce n'est pas un conte de fées. Enfin pas encore, ou rarement lorsqu'on étudie l'idéologie officielle d'un pays et les projets du peuplement blanc en Afrique. Une grande partie de ce travail minutieux est fondée sur les statistiques disponibles, et cette démarche naturelle avait déjà été suivie par les prédécesseurs de l'auteure. Mais elle va beaucoup plus loin qu'eux dans son analyse des origines sociales et géographiques, les âges, le rôle de l'État et des entreprises, etc. C'est une mine de données où l'on apprend, incidemment, les tentatives effectuées par quelques promoteurs juifs allemands pour installer en Angola et au Mozambique des milliers de colons en 1934, 1937 et 1938. Refus des autorités qui veulent des paysans portugais. La troisième partie est la plus originale puisqu'elle traite des relations entre les colons et leur nouvel environnement, les Africains et les Indiens (au Mozambique). Pas du tout naifs sont les chapitres sur les barrières raciales, la discrimination, les violences, le travail forcé, les cultures obligatoires, les évictions foncières, le traitement des indígenas par les commerçants, l'attitude vis-à-vis de l'Administration, la Métropole, l'Armée et la police. Cette partie est largement fondée sur des témoignages oraux ou publiés. Les comparaisons avec la colonisation blanche en Afrique noire (Kénya, Rhodésie) et en Algérie sont éclairantes,

${ }^{3}$ Cadernos de Estudos Africanos, Lisbonne, Instituto Superior de Ciências do Trabalho e da Empresa, Centro de Estudos Africanos, sans date d'impression, 5/6, 208 p. ; 7/8, 246 p. ; 9/10, 167 p.

${ }^{4}$ C. Castelo, Passagens para África. O povoamento de Angola e Moçambique com naturais da Metrópole (1920-1974), Porto, Edições Afrontamento, 2007, 405 p., graphiques noir et blanc et 2 cartes dépliantes en couleur. 
et les différences entre Angola et Mozambique sont également rappelées. Ce n'est pas une mise en accusation à la Basil Davidson ou à la Gerald Bender. Mesurée dans ses jugements, l'auteure pencherait plutôt vers notre «idéal » en histoire : donner des chiffres, exposer les faits, même les plus déplaisants, et laisser le lecteur tirer ses propres conclusions ( $c f$. R. Pélissier, La colonie du Minotaure. Nationalismes et révoltes en Angola, 1926-1961, Orgeval, France, Éditions Pélissier, 1979, 727 p.). La bibliographie est très sérieuse et irremplaçable pour la documentation d'origine officielle. Bref, c'est du beau travail.

Une chose est sûre avec Oil and Politics in the Gulf of Guinea ${ }^{5}$ de Ricardo Soares de Oliveira : ni l'innocence ni la naïveté n'ont jamais pointé le bout de leur nez dans les recherches de l'auteur ni, évidemment, dans leur objet. C'est un travail analytique d'un comparatiste redoutable qui connaît les rouages de l'industrie pétro-gazière dans les États rentiers qui vivent de leurs ressources minérales. Il maîtrise également bien l'étude des dommages sociopolitiques qui découlent de cette « huile du diable » dans des sociétés nullement préparées à les endiguer, à défaut de pouvoir les éliminer. Pour l'Angola, il rappelle le rôle politique des généraux d'une Armée surdimensionnée (110 000 hommes en 2004 et un tiers des dépenses de l'État), la cooptation des leaders de l'opposition par la corruption de marionnettes avides et l'intouchabilité des compagnies pétrolières. Il reconnaît cependant le professionnalisme de la Sonangol. Il n'accorde pas moins de dix pages à l'impuissance des Petits Poucets de São Tomé face à des prédateurs nigérians ou nord-américains. Selon certaines sources, l'expertise des gens de la Sonangol pourrait venir au secours de leurs cousins insulaires.

Pour la Guinée équatoriale, dix autres pages lui suffisent pour décrire la saga pétrolière du pays, face aux requins; c'est un État notoirement connu pour la judicieuse utilisation de ses royalties au profit de ses habitants les plus pauvres. Prenons l'exemple d'une de ses parcelles les plus modestes. D'après un missionnaire de l'époque, l'île de Corisco (située en plein dans une zone disputée avec le Gabon car supposée pétrolifère) comptait encore environ quatre cent cinquante habitants en 1943. Plus de soixante ans plus tard, on en trouve à peine cent cinquante (avant l'arrivée des pétroliers), selon un métis (dont la mère benga était originaire de l'île). Enfant, il y fut caché plusieurs années pendant que sévissait la folie paranoïaque de Papa Macías. Devenu citoyen espagnol et américain, Emilio Fontan Besay $^{6}$ est un exilé qui a réussi aux États-Unis où il est maintenant mannequin et acteur, mais il se souvient de ceux restés en arrière. De ce fait, il vient de publier l'un des rarissimes - très probablement l'unique - album de photographies sur cette terre qui autrefois portait le surnom de "la isla del amor» et qui le méritait bien, jusque dans les années 1960, malgré la présence des ruines encore imposantes d'une tête de pont catholique, disproportionnée par rapport aux $15 \mathrm{~km}^{2}$ de l'île. L'auteur est donc l'illustrateur d'une niche écologique, quasi oubliée des puissants mais plus pour longtemps, à mesure que les forages se rapprochent. De lusophone, elle n'a plus que son nom : Corisco.

${ }^{5}$ R. Soares de Oliveira, Oil and Politics in the Gulf of Guinea, Londres, C. Hurst \& Co, 2007, xiv-379 p.

${ }^{6}$ E.F. Besay, The Island of Corisco, [Philadelphia (Pa), États-Unis], Xlibris, 2007, 120 p., une centaine de photos couleur. 
Ne quittons pas les publications couvrant plusieurs pays sans mentionner, pour les amateurs de curiosités, un texte en tchèque de Jan Klíma ${ }^{7}$ qui résume à l'intention de ses compatriotes l'histoire de la colonisation - avec de longs développements ultérieurs sur la situation depuis l'indépendance - au Cap-Vert et à São Tomé et Príncipe. Il ne prétend pas être original, mais utile. Néanmoins, dans une section spéciale, il donne des indications précieuses sur les Tchèques qui ont visité et décrit le Cap-Vert, en particulier à propos des sokols à implanter dans l'archipel. Parfois il va loin dans les détails, notamment pour São Tomé entre 1960 et 1974. On suppose que les abondantes bibliographies en portugais qu'il cite sont totalement introuvables en Tchéquie.

Pour l'Angola, j'avoue mon incompétence et mon incapacité lorsqu'il s'agit de présenter le travail ${ }^{8}$ d'un professeur d'économie angolais ayant occupé de hautes fonctions dans l'Administration locale. Son analyse et sa théorisation du retard africain et de la corruption entraînée par cette situation montrent qu'il a vécu le problème de l'intérieur. Toutefois, il ne met pas le cas angolais spécialement en vedette. C'est peut-être plus sage, encore que la complexité du thème et du langage le place à l'abri des inquisiteurs trop curieux. Ce n'est pas un livre pouvant être compris par le premier ninja venu, ni même par le célèbre Jaime Bunda.

Facilement accessible et donc recommandable, un ouvrage, publié sous la direction de Patrick Chabal et Nuno Vidal, n'y va pas par quatre chemins. Je ne sais pas si tous ses auteurs obtiennent facilement leurs visas d'entrée dans le pays, ni même s'ils le demandent. Ce qui est clair, c'est que la flagornerie n'est pas leur défaut principal. Essentiellement politiques, les contributions dressent un tableau totalement carbonisé de la situation depuis l'indépendance. Dès l'introduction de Chabal, la tonalité est donnée. Selon lui, l'opposition n'a que peu de chances d'accéder au pouvoir par des élections libres tant que le Mouvement populaire de libération de l'Angola (MPLA) n'aura pas desserré son étreinte. L'Union pour l'indépendance totale de l'Angola (Unita) n'est plus qu'une façade de politiciens stipendiés, selon une autre contribution où l'on enregistre l'irrésistible concentration des pouvoirs dans les mains du président. On compare même ce dernier à un super-Machiavel, plus puissant que Salazar à son apogée. On est là dans le crime de lèse-majesté le plus caractérisé. Et ces "muckrackers» de politologues qui vous rappellent impunément que cette concentration vertigineuse avait commencé par Neto avant même le coup avorté de Nito Alves en 1977. Simple péripétie de quelques dizaines de milliers de morts. Il n’y a plus de façade doctrinale ? La belle affaire puisque le pétrole achète tout, à commencer par le Parti dont le président décide seul de l'orientation, de la composition et de l'évolution des effectifs qui, toujours d'après un auteur, passent de 65362 membres en 1990, à 544639 en 1992 puis à 2000000 en 2006, dont environ 1000000 à Luanda, ce qui est encore très peu. Selon lui, l'opposition serait sans moyens et sans cohésion (125 partis enregistrés en 2006) et d'ailleurs elle est financée par l'État, les chefs traditionnels, eux, étant aussi corrompus que l'appareil ou la «Justice».

7 J. Klíma, Kapverdské ostrovy. Svaty Tomás a Princuv ostrov, Prague, Libri, 2008, 179 p.

${ }^{8}$ E. Moreira CARneiro, Le blocage historique des économies africaines. Spécialisation rentière et extraversion, Paris, L'Harmattan, 2008, 218 p. 
Ces angolanistes étrangers nourrissent donc de bien noirs desseins et Charles Boxer avait finalement raison de ne pas dépasser 1825 dans son club de gentlemen. «Après 1825, c'est du journalisme », me disait-il. Simple admirateur du grand homme, vulgaire historien de la conquête de l'Angola, puis-je néanmoins oser faire une suggestion aux autorités? Pourquoi dépenser votre argent à organiser des élections en 2008 (à supposer qu'elles n'auront pas été repoussées)? C'est gâcher du papier à imprimer autant de bulletins de vote. É para Boxer ver? On doit pleurer dans les chaumières en Suède, chez les plus de 60 ans. Bref, cet Angola ${ }^{9}$, publié à Londres, sent le soufre et même quelque diablerie.

Et puisque nous sommes aux limites de la sorcellerie, franchissons les carrément et entrons dans l'occulte et le spirituel pour nous changer. $\mathcal{N} d o k i^{10}$ est un roman ethnologique (si l'on peut utiliser cette classification) d'un pasteur canadien qui a passé les années 1959-1997 chez les Bakongo, tant au Congo portugais/angolais qu'au Zaïre (avec les réfugiés de la guerre coloniale puis civile). Il témoigne d'une profonde empathie pour les Bakongo dont il maitrise la langue et les coutumes. Or celles-ci sont dominées par la crainte des sorciers, ce qui semble admis par tous les missionnaires connaisseurs du monde rural et des détribalisés de la région. L'histoire est complexe et peut se résumer ainsi : prise de possession d'une jeune femme puis son émancipation ou délivrance lorsqu'elle se range définitivement sous la bannière des baptistes (lesquels convertis baptistes furent d'ailleurs au premier rang des massacreurs de 1961 sur ordre de l'Union des populations d'Angola (UPA) de Holden Roberto et de ses apprentis sorciers/guérilleros). Ce qui nous intéresse ici, c'est le «placage» des facteurs et des activités des protagonistes «extérieurs ». Apparaissent donc les militaires portugais, les pressions coloniales, les combats entre le Front national de libération de l'Angola (FNLA), le MPLA (et les Cubains), l'Unita, les mines, la fuite vers la frontière, la guérilla de l'Unita, la vie des exilés au nord, l'influence des pasteurs tant blancs que noirs de l'Église baptiste, etc. En une trentaine d'années de contacts quotidiens avec ses ouailles (sincères ou intéressées), il en a appris des choses, notre auteur. La couleur locale nul besoin chez lui de la rechercher dans les livres des autres.

Les missionnaires en Angola rencontrent-ils plus de sorciers qu'ailleurs? C'est fort possible puisqu'on en retrouve un autre chez les Quiocos de l'Est angolais, dans une mission adventiste (Missão da Luz, nord-ouest de Luena/Luso), dans les années 1950. Mais il ne résista pas longtemps à la parole de Jésus. C'est ce que nous dit Lícius Lindquist ${ }^{11}$ reproduisant les souvenirs fort intéressants d'un vieux pasteur autrefois en activité (Benguela, Est, puis chez les Ovimbundu, et ensuite Luanda). Ses collègues et lui n'ont pas dû chômer puisque entre 1947 et 1974, le nombre de convertis passe de 400 à 45000 et, malgré Savimbi qui leur détruisit deux missions et leur hôpital et chassa leur grand chirurgien thaumaturge, les effectifs sont actuellement de plus de 200 000. Anecdotique mais fort lisible,

${ }^{9}$ P. Chabal \& N. Vidal (eds), Angola. The Weight of History, Londres, Hurst Publishers, 2007, $\mathrm{X}-246 \mathrm{p}$.

${ }^{10}$ Ch. H. Harvey, Ndoki. Trapped in the Web of Witchcraft, [Longwood (Fl), États-Unis], Xulon Press, 2007, 361 p.

${ }^{11}$ L. Lindquist, Aventuras do missionário Manuel de Castro : entre os leões de Angola, Tatuí (SP, Brésil), Casa Publicadora Brasileira, 2006, 144 p. 
ce petit livre nous «promène » de l'Angola colonial des profondeurs à l'horreur de la guerre en brousse.

Depuis l'indépendance des Palop, on note une forte offensive des missionnaires brésiliens vers un champ rétréci, abandonné, difficile ou qui fut longtemps interdit lorsqu'on était portugais ou nord-américain. Surtout dans un pays en guerre comme l'Angola de 1992 où revient la missionnaire Antonia Leonora van der Meer ${ }^{12}$ qui débarque en octobre à Luanda en plein chaos. C'est une intrépide (mais malade) qui nous offre environ deux centaines de pages sur ses tribulations d'une dizaine d'années en Angola, à partir de Luanda en 1984. Dans le maquis des Églises protestantes, on se perd un peu, et de plus elle déploie une activité ennemie de la sédentarité, sautant de Lubango à Huambo, Sumbe, au Mozambique, à Luanda, etc. Cela ne facilite pas le récit, mais comme elle aborde des sujets peu traités (bourses brésiliennes pour les étudiants, évacuation des résidents étrangers en 1992, jeunesse masculine réfractaire au service militaire, voyages sur les routes minées, résistance (?) des soldats cuanhama à la corruption ambiante, cartes de rationnement alimentaire, cambriolages, relations avec les pasteurs locaux méfiants, aumônerie dans les hôpitaux "dantesques » de réhabilitation des blessés de guerre, les mutilés, etc.), son témoignage a une valeur documentaire indéniable pour connaître l'Angola paroxystique, autrement qu'à travers les journalistes extérieurs ou les humanitaires de passage, tombant sans préparation dans cette horreur.

Horreur qu'un jeune tétraplégique kongo, victime d'un accident, nous fait découvrir en soulevant un tout petit coin du voile puisqu'il ne concerne qu'un hôpital de Luanda où il fut interné pendant sept ans et dix mois. Dans un pays où l'on parle officiellement mais vaguement de 150000 mutilés de guerre, il y a longtemps que l'innocence a déserté. Sa déposition ${ }^{13}$ a été rédigée avec un stylo dans la bouche. Encore lui s'en est relativement bien sorti, pris en mains par l'Église à laquelle appartient la précédente auteure. Pour les autres... Probablement qu'ils attendent les élections.

Donc, on est dans la guerre civile, mais revenons en arrière, vers celle des Portugais entre 1961 et $1974-75$, avec un livre ${ }^{14}$ curieux d'un grand-père, journaliste, membre du PS, très corpulent mais ayant le sens de l'humour et du style, ce qui se raréfie dans la littérature des anciens combattants, même de droite. Ce n'est pas un roman, ni un témoignage, ni un poème, ni un traité savant. Il n'est pas non plus daté, ni vraiment localisé. C'est une succession de petites histoires qui paraissent se dérouler dans les années 1960 (vers le milieu ou la fin ?) entre Luanda et le Nord-Ouest, du côté de Nambuangongo, dans les Dembos, mais on va aussi sur la côte. La force du texte est qu'il sautille entre trente-deux vignettes où l'on voit ce qu'étaient les soldats d'une garnison (?) portugaise dans la jungle, ainsi que les maquisards du MPLA, leurs biographies, leur misère, leur étonnement d'avoir à se combattre. C'est, naturellement, un livre antisalazariste, mais aussi

12 A.L. van der Meer, Eu, um missionário? Quando o jovem cristão leva a sério o seu chamado, Viçosa (MG, Brésil), Editora Ultimato - São Paulo (SP, Brésil), ABU Editora, 2006, 238 p.

${ }^{13}$ J. Gomes, Da tragédia para Cristo. Da agonia à coragem para lutar. Do ateísmo à coragem para crer, Viçosa (MG, Brésil), Editora Ultimato, 1998, 183 p.

${ }^{14}$ A. Ferreira, Morte na picada, Lisbonne, Via Occidentalis Editora, 2008, 238 p., photos noir et blanc. 
antimilitariste et même peut-être antinationaliste. Il faut un dictionnaire qui n'existe pas pour traduire tous les mots du vocabulaire utilisé. L'auteur ne travaille pas dans la fresque monumentale, mais plutôt dans le genre "vitrail caméléonesque » et réaliste. Pas d'épanchements psychologiques : du vécu mal vécu pendant deux ans. Et cela finit mal. Donc à recommander au lecteur linguistiquement bien armé pour comprendre l'argot en usage à l'époque en Angola, de part et d'autre de la piste de la mort.

Le gros livre qui suit possède un titre ${ }^{15}$ qui m'a trompé. Je croyais que l'auteure, pédopsychiatre de son état, allait nous parler de la récupération mentale des petits enfants angolais broyés par des années de guerre civile, et de leurs expériences entre les maquis de l'Unita, les villes du Centre bombardées et détruites, et finalement les camps de deslocados. Totale erreur de ma part. En fait, il s'agit bien de souvenirs d'enfance et d'adolescence, mais ce sont ceux de l'auteure qui, née en 1932, dans la concession diamantifère de la Lunda, de parents blancs (mais nés en Inde) où le mari est un chefe de posto, puis devient un administrateur et grimpe plus haut encore, au fil de sa carrière. Comme il défendra les droits des Africains - apparemment, ceux qui les bafouaient n'ont pas laissé beaucoup de livres pour s'en vanter - il fut en butte à l'hostilité de sa hiérarchie. Étant trop petite pour se remémorer ses toutes premières années, elle accumule les données ethnologiques sur les populations, ce qui n'est pas vraiment l'objet du texte. Son intérêt commence donc quand, entre 1935 et 1938, elle s'installe à Cuangar, via un long voyage en tipoia et char boer. À la frontière du Sud-Ouest africain, c'est l'un des pôles d'inaccessibilité coloniale de l'époque: un seul commerçant, pas d'école, la mère fait tout (habits, meubles, etc.). Elle visite les missions allemandes et l'administrateur sud-africain de la rive droite du Cubango/Okavango. Le paradis cesse lorsque le père est muté à Caala (1939-41). Là, les colons utilisent le travail forcé des contratados, d'où l'opposition du chef de famille, un légaliste. Intrigues et pressions : on le transfère à São Salvador (1941-42) où il ne fera pas long feu non plus car, anticlérical, il fréquente la mission baptiste, les curés courant les villages pour satisfaire leurs besoins sexuels, et le roi du Congo, Dom Pedro VII, étant une simple potiche décorative. Déjà plus grande, elle peut décrire des postes éloignés comme Noqui, Cuima, etc.

Ce sont les cinq dernières années (1942-47), dans la colonie pénale pour «droits communs " de Porto Alexandre, puis à Moçâmedes où le père est intendente, qui fournissent le plus de détails sur la vie des colons et des Africains (dont les condamnés qui circulent en liberté, le désert empêchant toute fuite). L'auteure a eu raison d'illustrer le livre avec de très nombreuses photos montrant le quotidien d'une famille dans ses années déjà lointaines.

Et puisque nous sommes presque arrivés au sommet du cycle colonial qui allait s'achever dans la débandade de 1974-75, faisons retour sur les mythes et la mémoire collective. Qu'un roman intitulé Os retornados. Um amor nunca se esquece $e^{16}$ se vend au Portugal à plus de 12000 exemplaires en deux semaines, entre février

${ }_{15}$ M.J. Vidigal, Um olhar de criança sobre Angola. Grandes e pequenas histórias de uma Angola imensa, Lisbonne, Trilhos Editora, 2007, 311 p., photos noir et blanc et couleur.

16 J. Magalhães, Os retornados. Um amor nunca se esquece, Lisbonne, A Esfera dos livros, 2008, 309 p., photos noir et blanc. 
et mars 2008, est un symptôme qui en dit long, même si, probablement, l'auteur, journaliste de la télévision, a dû bénéficier d'une promotion confraternelle et éditoriale fort efficace. Ce n'est pas un mauvais livre, loin de là, mais le serait-il, que le thème de la fuite ou expulsion de centaines de milliers de colons d'Angola dans des conditions déshonorantes pour les "pouvoirs» en place à Lisbonne et à Luanda (sans rien dire de Lourenço Marques/Maputo) est suffisamment gravé dans la mémoire d'une génération (et même de celle qui la suit) au Portugal pour que cette fin douloureuse de l'Império ait une importance comparable à l'Alcácer Quibir du roi Sébastien. On ne va pas se lancer ici dans l'une de ces gloses pseudo-psychanalytiques dont raffolent philosophes et littéraires universitaires, mais il est clair dans l'esprit de nombreux historiens - et probablement dans l'esprit de ceux qui ont acheté le livre - que cet amour inoubliable qui existait et existe, consciemment ou non, s'adresse à l'Ultramar avec une intensité que l'on ne retrouve dans aucune autre métropole européenne. Un amour qui ne veut voir ni verrues, ni rides, ni sang, pour ne retenir que le rêve de sa grandeur et de son unicité. On n'échappe jamais à son école primaire et maintenant que le ressac politique commence à s'estomper avec la disparition inéluctable des protagonistes, il est à parier que l'Ultramar perdu va prendre une importance croissante dans les préoccupations des lettrés au Portugal au cours des années à venir. En tant que spécialiste je m'en réjouis, en tant que bibliographe accablé de travail beaucoup moins.

Exploitons outrageusement le fil de l'éternel retour impérial pour glisser encore ici le livret ${ }^{17}$ d'accompagnement d'une exposition (6 décembre 2007-31 décembre 2010) qui n'a rien à voir avec les colons portugais puisqu'il s'agit de présenter la deuxième mission scientifique suisse en Angola (avril-novembre 1933), le conservateur Théodore Delachaux et les 3500 objets qu'il acheta - pas cher - du Centre-Angola (Bimbe) à l'extrême-sud (Ruacana et pays ovambo). Cela intéressera les ethnologues et les muséographes spécialistes de l'Angola. Rien sur l'Histoire.

Et maintenant le Mozambique, en commençant par une vision brésilienne. Un beau titre, assurément, De escravo a cozinheiro ${ }^{18}$ est essentiellement une histoire du travail auquel le système colonial portugais soumit les Africains du SudMozambique. Elle s'adresse en priorité à un public brésilien peu au fait de la littérature scientifique relative au thème. Les travaux bien connus de Jeanne Penvenne ne doivent probablement pas se vendre au coin des rues à Bahia. De ce fait, la bibliographie est abondante mais inégale : le général colonialiste Teixeira Botelho y figure, mais aucune des "História de Moçambique " plus récentes, traduites en portugais, ni même le chef-d'œuvre de Vail et White en anglais sur le BasZambèze, qui aurait pu offrir des points de comparaison. C'est néanmoins une thèse sérieuse qui analyse systématiquement les mécanismes mis en œuvre pour faire produire un maximum à moindres coûts - impôts, expropriations foncières, travail forcé ou « volontaire », racisme dans l'emploi, inégalité des salaires et même

${ }^{17}$ Collectif, Retour d'Angola, Neuchâtel (Suisse), Musée d'ethnographie, 2007, 80 p., photos et dessins noir et blanc.

18 V. Zamparoni, De escravo a cozinheiro. Colonialismo e racismo em Moçambique, Salvador (Brésil), Editora da Universidade Federal da Bahia-Centro de Estudos Afro-Orientais, 2007, 338 p., photos noir et blanc. 
dans la répression des grèves -, les verrues de l'Império que certains ne veulent pas se rappeler et d'autres ne pas voir.

Plus technique encore, un recueil d'études approfondies ${ }^{19}$ consacrées aux élections des institutions municipales, à leurs pouvoirs et à leurs activités après la décentralisation, intéressera ceux qui ont connu Inhambane, Vilanculo, Dondo, Chimoio, Moçambique (l'île), Montepuez. Très fouillées, très libres d'appréciation, ces micromonographies témoignent de la vigueur d'un esprit critique à l'égard du Front de libération du Mozambique (Frelimo) et de la Résistance nationale mozambicaine (Renamo), c'est-à-dire d'un renforcement de la «démocratie », malgré les difficultés, les contraintes, les pressions et le poids écrasant des traditions. C'est la Coopération suisse qui a financé l'enquête.

Dans le même ordre de préoccupations, signalons la parution, sous la direction d'Elísio Salvado Macamo, de l'une de ces innombrables juxtapositions qui prolifèrent dans les sciences politiques du monde anglo-américain. Negotiating Modernity ${ }^{20}$ comporte trois contributions d'auteurs mozambicains examinant, sous un angle historique et surtout sociologique, comment les habitants du Sud-Mozambique, influencés par les missions protestantes et l'émigration vers l'Afrique du Sud, s'approprièrent certains éléments de la modernité, malgré le corset colonial portugais. Il n'y a pas de surprises : dénonciation argumentée du colonialisme, de l'indigénat et de la prétendue apathie des Africains. Parmi les textes les plus intéressants, on note celui relatif aux mineurs convertis par les Anglicans, devenant des évangélistes au Mozambique. Un autre porte sur l'émigration rurale vers Xai-Xai (Gaza) par suite de la guerre civile et des inondations. Tout cela commence à être bien connu et il serait peut-être temps de monter vers le Nord, maintenant.

Toujours à la pointe de la mode des dénonciations universitaires, marxisantes, féministes et virulentes, un nouveau festival ${ }^{21}$ antimondialiste et tiers-mondiste? "No problem »: il suffit d'étendre le bras et une contribution toute " fraîche » - mais fort bien documentée - tombe sur votre table. Son auteure arrive même à nous convaincre que le Frelimo, première manière, doit être exonéré de toute responsabilité dans l'état calamiteux du pays. L'ultra-pauvreté est la faute exclusive, selon elle, du néolibéralisme. Elle insiste - à bon droit - sur le rôle de la presse protestataire dans la révélation d'une corruption et d'une violence insupportables. Et pour elle, ces tares sont nées des dénationalisations dans les secteurs productifs et les services publics. Pourquoi pas, au point où nous en sommes?

Quittons ces sentiers battus et rebattus pour aller vers des auteurs qui, le monolinguisme prévalant désormais dans le monde anglo-américain et ses satellites lusophones, ne risquent pas d'aérer les bibliographies altermondialistes. Le Tchèque Jan Klíma ${ }^{22}$, encore lui, récidive avec une monographie qui résume en détail ce qui est établi en anglais, français et portugais sur le Mozambique. Curieusement,

${ }^{19}$ L. de Brito, E. Maguacua, F. Kulipossa, N. Ernesto, Partidos, cidadãos e eleições locais em Moçambique - 2003, Maputo, Imprensa Universitária, 2005, 205 p.

${ }^{20}$ E.S. Macamo, Negotiating Modernity. Africa's ambivalent experience, Londres, Zed Books, 2005, $\mathrm{V}-238$ p.

${ }^{21}$ G. Eschle \& B. Maiguashca (eds), Critical Theories, International Relations and "the AntiGlobalisation Movement». The Politics of Global Resistance, Abingdon (Angleterre), Routledge, 2005, XVIII-264 p., photos noir et blanc.

${ }^{22}$ J. KLÍma, Mosambik, Prague, Libri, 2007, 182 p. 
il expose avec beaucoup de soins la Grande Guerre au Mozambique. Serait-ce notre mauvaise influence qui l'aurait contaminé? Le nationalisme, la guerre coloniale, l'indépendance marxiste, la guerre civile, l'évolution depuis sa cessation et les relations historiques avec son pays occupent une soixantaine de pages. J'avoue ne pas avoir bien compris ce que le Dr Emil Holub a à voir avec le Mozambique, mais Klíma fait le maximum pour trouver des liens, si ténus soient-ils.

En revanche, les Italiens, tant missionnaires que coopérants ou humanitaires, tiennent une grande place au Mozambique et n'ont pas à racler les fonds de tiroir pour occuper une part croissante de la bibliographie mozambicaniste. À cheval sur les trois tableaux, le Dr Antonio Scisci nous livre ses impressions ${ }^{23}$, à l'issue d'une mission d'un mois (juin-juillet 2006) où il vient aider les missionnaires italiens en Basse-Zambézie (Quelimane, Mopeia, etc.). Il est surtout utile pour connaitre le quotidien des malades (sida, paludisme, etc.), les enfants des rues et la pauvreté en général («l'enfer de Maputo » qui l'épouvante). Il voyage beaucoup entre ses consultations et fournit force détails sur l'œuvre caritative de ses compatriotes. En fait, son livre est surtout un appel à la générosité des catholiques italiens pour qu'ils aident ces missionnaires écrasés par l'ampleur et, peut-être, la dualité de leur tâche.

Voici maintenant un professionnel à plein-temps de la charité internationale où il semble exorciser son mal de vivre en courant les pays en guerre, à l'ombre ou sous contrat des Nations unies et d'autres bienfaiteurs de l'humanité. Pour son livre de confessions morbides, apparemment libératrices, on se limitera au petit chapitre mozambicain où, à peine sorti du service militaire, ce jeune Sicilien est chargé, à partir de Beira, de contacter les troupes de la Renamo pour les concentrer dans des camps de regroupement montés par les Nations unies, à l'issue de la guerre civile. On en apprend beaucoup sur la vie dorée des diplomates italiens (13 000 dollars mensuels, au minimum, à Maputo dans ces années-là où le salaire d'un boy était au maximum de 40 dollars!). Lui commence par les bases de la rébellion en pleine brousse. Fasciné par son premier village «à la Conrad» où survivent les mutilés de la guérilla et quelques combattants tombés d'un cadre de Jérôme Bosch, il n’a pas trop de mal à nous dépeindre le chaos post-traumatique de ces deux années (1992-94) de sortie de guerre, entre les jouissances du surhomme pour les Blancs (civils ou militaires) et la déshumanisation pour les Noirs rescapés. Il trouve même le moyen de montrer un trafic de peaux de lions et d'ivoire d'hippopotame sous le couvert de l'ONU et de son programme alimentaire. Avec Giorgio Trombatore ${ }^{24}$, on est toujours très loin de la bonne conscience et de la suffisance des fonctionnaires rédigeant leurs rapports à l'eau de rose. On se demande même s'il est nécessaire de citer Nietzsche dans ces cas-là, quand on fréquente cette faune bouffie de bienveillance. Rémunératrice si possible.

Dans le genre poignant, on peut difficilement faire mieux que de conseiller le livre de Marijke Teeuw ${ }^{25}$ qui, contrairement au Suédois Mankell, ne donne pas dans la fiction mais dans le document authentique. Envoyée en 1989 par une

${ }^{23}$ A. Scisci, Medico volontario in Mozambico. Le missioni francescane, Foggia (Italie), Editrice Parnasso, 2007, 255 p., très nombreuses photos couleur.

${ }^{24}$ G. Trombatore, Coy Ecce Homo. Storie di un operatore umanitario, Catane, Le Nove Muse Editrice, 2008, 202 p.

${ }^{25}$ M. Teeuw, Moisés, kind van Mozambique, Eindhoven (Pays-Bas), Uitgeverij Pepijn, 2008, 411 p. 
organisation d'aide au développement au Mozambique, elle y recueille un enfant des rues, un orphelin de douze ans, le soigne, le suit, le scolarise, etc. En fait, ce long récit est une plongée vertigineuse dans la société mozambicaine jusqu'en 2000, date du retour de l'auteure aux Pays-Bas. Onze ans de séjour dans les rouages de l'Administration, au cœur des familles africaines, des écoles, des milieux humanitaires, des expatriés, des Églises, etc. donnent une vision pénétrante des réalités du pays, de Nampula à Maputo. Elle va, avant son départ, jusqu'à construire une maison pour son protégé. Mais celui-ci, devenu adulte, tourne mal. Six mois après que la bonne fée a disparu en reprenant pied en Europe, il est assassiné. On n'ose ajouter que c'est une parabole pour prédire le destin d'un pays que l'auteure a aimé autant que son pupille. Ce serait du pessimisme, mais il est à craindre qu'elle ne s'en relèvera pas de sitôt.

On le sait ou on ne le sait pas, mais le Mozambique est le seul pays africain à avoir eu des contacts intermittents avec Timor, d'abord militaires, sous le manteau impérial portugais, puis politiques après l'indépendance. Cela nous donne un mince prétexte pour enchaîner maladroitement avec le dernier maillon oriental de la Communauté des pays de langue portugaise.

Trois livres dont le plus intéressant est le dernier. On commencera par celui du père Francisco Maria Fernandes ${ }^{26}$, prêtre timorien qui a consacré sa dissertação de mestrado au premier évêque de Timor qui, depuis Macao, relança l'évangélisation du Timor portugais à la fin du $\mathrm{xIx}^{\mathrm{e}}$ siècle. C'est le travail modeste d'un amateur sur le plan strictement historiographique. Il ne possède nullement la bibliographie pertinente et occulte tout ce qui pourrait nuire à l'image de son héros, notamment les dissensions avec son cousin, le terrible gouverneur Celestino da Silva. C'est de l'hagiographie aux frontières de l'angélisme et du remplissage (à peine quelques dizaines de pages concernent réellement l'évêque et son rôle fort important). La meilleure chose? La reproduction des pages que lui a fournies le maître compilateur des périodiques publiés à Macao à l'époque considérée, le minutieux Kevin Sherlock.

Le Timor oriental face à la Seconde Guerre mondiale (1941-1945) est un titre alléchant a priori. Malheureusement, il est trop ample et ce mémoire de licence, soutenu à Genève par un Luso-Suisse, est centré presque uniquement sur le contexte diplomatique. Là, l'auteur est très utile car il a travaillé aux archives du ministère portugais des Affaires étrangères et il est probablement le premier à l'avoir fait pour cette période et ce thème. Mais il n'y a que très peu de chose sur les opérations militaires et la vie pendant l'occupation japonaise, alors que c'était crucial et que les témoignages portugais et australiens à ce sujet sont multiples. Certaines traductions du portugais sont également flottantes ou incomplètes.

Finalement, ce qui nous informe le mieux, on le doit à une Brésilienne pétulante, professeure de journalisme, de publicité, de lettres et d'art dramatique (c'est tout un pour cette rosicrucienne, adepte également de l'Énergie cosmique supérieure,

${ }^{26}$ F.M. Fernandes, D. António Joaquim de Medeiros (Bispo de Macau) e as Missões de Timor, 18841897, Macao, Gentro de Publicações, Universidade de Macau, 2000, 76 p. et ensuite une centaine de pages reproduisant des articles d'auteurs divers et des documents en photocopies non paginées, photos noir et blanc.

27 F.B. D’ÁGuA, Le Timor oriental face à la Seconde Guerre mondiale (1941-1945), Lisbonne, Instituto Diplomático, 2007, 263 p., photos noir et blanc. 
du bouddhisme et d'autres voies vers la Lumière intérieure), membre d'un groupe de cinquante coopérants envoyés à Timor par Brasília en mai 2005. Comment ils ont été recrutés restera pour le lecteur un nouveau mystère de la lusophonie, puisque certains croyaient encore que Timor se situait en Afrique! C'est donc un macro-reportage ${ }^{28}$ scindé en plusieurs dizaines de scènes parfois désopilantes, mais devenant dramatiques à la fin.

Elle touche un peu à tous les genres, n'est pas toujours fiable dans la chronologie et les statistiques, mais lorsqu'on aborde 1) les dissensions ethniques entre les soldats timoriens en 2006 puis les émeutes qui s'ensuivirent en avril de cette année-là ; 2) la fuite des habitants de Dili, la peur et l'évacuation des expatriés, l'arrivée des renforts australiens; 3) l'incendie de centaines de maisons, l'exfiltration des Brésiliens vers Darwin (mais certains, dont l'auteure, reviennent à Dili s'occuper des camps de réfugiés : 100000 personnes que se disputent les ONG); 4) la bureaucratie locale et onusienne ; 5) la visite de certains quartiers incendiés et abandonnés; 6) le montage d'une pièce de théâtre (sic) pour apaiser (sic) les tensions ; 7) les attaques des gangs de jeunes qui tuent, décapitent et démembrent dans la ville leurs adversaires ethniques ; 8) la mise au pas provisoire de ces jeunes assassins par la Garde nationale républicaine (GNR) du contingent portugais (les seuls à faire front, dit-elle, alors que les Australiens et les Néo-Zélandais sont inefficaces) ; 9) l'enterrement du voisin tué, dont elle paie le cercueil, etc., l'on se dit alors que cette femme exaltée et ses Maîtres cosmiques qu'elle implore sur la plage valent peut-être mieux que les dizaines de milliers de soldats étrangers et savants experts en détresses qui ont défilé à Timor depuis 1999. Avec quels résultats?

Grandes espérances, maigre innocence et beaucoup de suffisance dans ce et ces pays désertés par les esprits du Bien et de la Sagesse.

Mai 2008, René Pélissier, GNRS 